\title{
Supervisión escolar y cambio educativo. Uruguay a la luz de la experiencia internacional (2005-2020)
}

\author{
School inspectors and educational change. The Uruguayan case \\ in light of the international experience (2005-2020)
}

\section{María Ester Mancebo* y Margarita Romero**}

* Doctora en Ciencias Humanas, opción Educación (Universidad Católica del Uruguay). Magíster en Ciencias Sociales, opción Ciencia Política (FLACSO Argentina). Profesora de Historia (IPA, Uruguay). Profesora titular D. T. en el Depto. de Ciencia Política (FCS, Udelar). Investigadora activa del Sistema Nacional de Investigadores de Uruguay (nivel II). $\bowtie$ memancebo@gmail.com https://orcid.org/0000-00029002-7930

** Doctora en Educación por la Universidad ORT Uruguay. Magíster en Ciencias de la Educación, opción Currículum y Evaluación, por la Universidad Católica del Uruguay. Profesora de Literatura y de Idioma Español (IPA, Uruguay). $\triangle$ margaritaromerogarcia@ gmail.com https://orcid.org/0000-00029343-9287

\section{Resumen}

Los inspectores constituyen un eslabón fundamental en todo proceso de transformación educativa. Este artículo se propone comprender su papel en las múltiples innovaciones implementadas en la educación primaria y secundaria de Uruguay durante la era progresista (2005-2020), período en que el gobierno nacional estuvo encabezado por un partido de centroizquierda, el Frente Amplio. El trabajo se nutrió de bibliografía sobre la inspección docente y sobre los procesos de cambio, con aportes provenientes de la pedagogía y la ciencia política. Se trata de una investigación descriptivoexplicativa, de carácter cualitativo y con una tónica comparativa. Como principales hallazgos se encontró que, bajo una misma institucionalidad, la postura de la Inspección de Primaria ante las innovaciones fue divergente respecto a la asumida por la Inspección de Secundaria. A nivel explicativo se encontró que el papel de la supervisión en la transformación educativa derivó no solo de la institucionalidad dominante, sino también del respaldo político, la modalidad de implementación de las políticas innovadoras, la cultura profesional prevaleciente y el peso de la historia.

Palabras clave: inspección educativa, política educacional, Uruguay 


\section{Abstract}

School supervisors constitute a fundamental link in any process of educational transformation. This article aims to understand its role in the multiple innovations implemented in Uruguay during the "progressive era» (2005-2020) in which the national government was led by a center-left party, the Frente Amplio. The article was nourished by bibliography on teachers' supervision and on change processes, with contributions from both Pedagogy and Political Science. The research carried on was descriptive and explanatory, qualitative in nature and with a comparative tone. As main findings, the document emphasizes that, under the same institutional framework, the position of Primary supervisors towards innovations diverged from that assumed by supervisors in the Secondary level. At an explanatory level, it was found that the role of supervision in educational transformation derives not only from the dominant institutional framework but also from political support, the modality of implementation of innovative policies, the prevailing professional culture and the weight of history.

Keywords: Educational supervision; Educational policy; Uruguay

\section{Introducción}

Este artículo estudia el rol de la inspección escolar en la transformación de la educación pública en Uruguay durante la llamada era progresista (2005-2020), período en el cual, por primera vez en su historia, el gobierno nacional estuvo en manos de un partido de centroizquierda, el Frente Amplio (FA). Uruguay es un país pequeño, unitario, y en el conjunto de la administración estatal ha mantenido un modelo weberiano. En educación, a lo largo de su historia, el país creó una fuerte tradición de estatismo, preservó el Estado docente y logró un desarrollo educativo avanzado en la región, no obstante lo cual en las últimas décadas el sistema educativo ha registrado indicadores preocupantes de repetición, rezago y no completitud de la educación media (Sistema de Información de Tendencias Educativas en América Latina [SITEAL], 2019; United Nations Educational, Scientific, and Cultural Organization [UNESCO], 2016).

El gobierno del FA no impulsó una reforma educativa integral, ya que la implementación de algunas de las transformaciones ineludibles ${ }^{1}$ hubiera representado altos costos políticos (Mancebo y Pérez, 2018). El gobierno del FA apostó a la expansión del acceso a los niveles educativos obligatorios (14 años en total), incrementó significativamente el presupuesto educativo e impulsó el desarrollo de múltiples programas de in-

1 Alteración del formato escolar, reforma del Estatuto Docente, modificación profunda de la formación inicial docente, para citar ejemplos neurálgicos. 
clusión que representaron innovaciones educativas interesantes (Bentancur y Mancebo, 2011). Como ejemplo de estas innovaciones, cabe citar el Programa de Maestros Comunitarios, el Programa Aprender y el Programa Tránsito Educativo en primaria, y el Programa de Impulso a la Universalización de la Educación Básica, el Programa de Aulas Comunitarias y el Programa Uruguay Estudia en secundaria. ${ }^{2}$

Estas innovaciones fueron aplicadas en pequeña escala (Instituto Nacional de Evaluación Educativa [INEEd], 2014) y supusieron leves transformaciones en los formatos pedagógicos y sus soportes organizacionales: tiempos pedagógicos extendidos, espacios de aprendizaje ampliados, modalidades diferentes a la del aula tradicional (clases a contraturno, talleres, estímulo al pensamiento lúdico, por ejemplo). También hubo una fuerte articulación con agentes de la comunidad y con instituciones no educativas, se apostó a fortalecer el trabajo en equipo de los docentes, se incentivó la ejecución de proyectos institucionales y el trabajo con las familias. Además, se crearon nuevas figuras pedagógicas (maestros y profesores comunitarios, tutores, profesores coordinadores pedagógicos, entre otros) y se dotó a los centros educativos de equipos multidisciplinarios de asesoramiento integrados por asistentes sociales y psicólogos.

Las investigaciones sobre estas innovaciones (Romero, 2018, 2011; Fernández y Mancebo, 2015; Moreno et al., 2014; Pérez, 2011; Fernández y Pereda, 2010) profundizaron en sus líneas de trabajo e instrumentos, su institucionalidad, sus resultados y su impacto. El papel de los supervisores en el desarrollo de estos procesos de transformación de la educación uruguaya no ha sido aún estudiado, y esto constituye el objeto central de este artículo, que se orienta por las siguientes preguntas:

- ¿Qué papel tuvieron los inspectores en los procesos de cambio educativo impulsados por el gobierno de la educación de Uruguay en el período 2005-2020?

- ¿Qué factores pesaron sobre el impulso o el freno de los inspectores a las innovaciones?

\section{Marco teórico}

\section{Los modelos de supervisión educativa}

La literatura especializada sobre supervisión educativa es copiosa: se han analizado las percepciones que poseen los docentes sobre ella (Bueno y Cababaro, 2019), la

Este estudio se concentró en innovaciones desarrolladas en el ámbito público en primaria y secundaria (Consejo de Educación Inicial y Primaria, CEIP, y Consejo de Educación Secundaria, CES); no se incluyeron las implementadas en la educación técnica (Consejo de Educación Técnico-Profesional, CETP). 
óptica de la formación para este rol (Rivera Hernández, 2017), se ha planteado la posible conversión de la supervisión de control en una instancia colaborativa y participativa (Sergiovanni y Starratt, 2007), se ha analizado su inserción en el sistema educativo y posible transformación (Zorrilla, 2013) y también se la ha criticado profundamente considerando sus acciones como inefectivas e ineficientes (Marshall, 2005).

Existen diversas clasificaciones de los modelos de supervisión, entre las que se destaca la elaborada por de Grauwe y Carron (2003), quienes identificaron cuatro «tipos ideales» con base en tres dimensiones: 1) el rol y los objetivos de la supervisión, 2) sus herramientas de monitoreo y 3) el tipo de responsabilidad de los docentes. La revisión bibliográfica realizada (Aguerrondo, 2013; Choi, 2019; Rivera Hernández, 2017; Canal Ventosa, 2018) permite incorporar un cuarto criterio: 4) la organización de la supervisión como unidad de la administración estatal, la que puede ser pensada en un continuo con dos polos: uno de tipo burocrático-tradicional ${ }^{3} \mathrm{y}$ el otro cercano al modelo de Nueva Gestión Pública. ${ }^{4}$ Los casos nacionales podrían ubicarse en distintos puntos del continuo (tabla 1 ).

Tabla 1. Tipología de modelos de supervisión docente

\begin{tabular}{|l|l|l|}
\hline Modelos & Países & Rasgos principales \\
\hline $\begin{array}{l}\text { Control } \\
\text { público }\end{array}$ & Francia & $\begin{array}{l}\text { La Inspección (Nacional y Regional) realiza una evaluación externa } \\
\text { de los centros educativos y los monitorea. } \\
\text { Los inspectores elaboran informes generales y regionales de eva- } \\
\text { luación del sistema educativo. } \\
\text { Fuerte énfasis en el derecho de cada docente y cada centro educa- } \\
\text { tivo a ser supervisados. } \\
\text { Los docentes asumen que son responsables ante su empleador, el } \\
\text { Ministerio de Educación Nacional. } \\
\text { Organización burocrático-tradicional. }\end{array}$ \\
\cline { 3 - 4 } & \multirow{2}{*}{ España } & $\begin{array}{l}\text { La Inspección realiza la evaluación de los centros educativos y los } \\
\text { docentes. } \\
\text { El Instituto Nacional de Evaluación Educativa diseña e implementa } \\
\text { pruebas estandarizadas. } \\
\text { Organización burocrático-tradicional. }\end{array}$ \\
\hline
\end{tabular}

3 El modelo de administración pública estatal (APT) se caracteriza por responder a una autoridad jerárquica claramente identificada, seguir rutinas y procedimientos preestablecidos y estandarizados, y mostrar apego a la legalidad de los procedimientos administrativos (la llamada cultura del expediente).

$4 \quad$ El modelo de nueva gestión pública (NGP) busca hacer la administración pública más eficiente a través del empleo de técnicas del sector privado, la creación de condiciones de mercado para la entrega de los servicios públicos, la flexibilización de los procesos administrativos, la fijación de medidas de rendimiento y la libertad de gestión para los gestores. 


\begin{tabular}{|c|c|c|}
\hline \multirow[t]{2}{*}{$\begin{array}{l}\text { Control } \\
\text { central }\end{array}$} & Inglaterra & $\begin{array}{l}\text { La Office for Standards in Education, Children's Services and Skills } \\
\text { (Ofsted), institución dependiente directamente del gobierno (no de } \\
\text { un ministerio en particular), tiene la función de inspeccionar el fun- } \\
\text { cionamiento de las escuelas (no a los docentes). } \\
\text { Las visitas de Ofsted a los centros se realizan cada tres o cinco años, } \\
\text { con diagnóstico de los resultados educativos y planteo de objetivos } \\
\text { de mejora. } \\
\text { Organización intermedia, abocada a la rendición de cuentas, con un } \\
\text { sistema jerárquico centrado en la evaluación de las escuelas y el } \\
\text { sistema educativo. }\end{array}$ \\
\hline & $\begin{array}{l}\text { Nueva Ze- } \\
\text { landa }\end{array}$ & $\begin{array}{l}\text { La Educational Review Office realiza evaluaciones externas, com- } \\
\text { plementarias a las autoevaluaciones institucionales. } \\
\text { Los inspectores o review officers son contratados para realizar la } \\
\text { evaluación externa; visitan las escuelas una vez cada tres años y sos- } \\
\text { tienen su presencia en aquellas que presentan mayores dificultades. } \\
\text { Los reportes realizados por los review officers, luego de aprobados, } \\
\text { se publican para los grupos interesados en la escuela y la educación. } \\
\text { Organización intermedia, promotora de procesos de evaluación au- } \\
\text { togestionados por los centros educativos y rasgos de NGP. }\end{array}$ \\
\hline $\begin{array}{l}\text { Apoyo } \\
\text { cercano } \\
\text { a la es- } \\
\text { cuela }\end{array}$ & Chile & $\begin{array}{l}\text { Los supervisores asesoran pedagógicamente a las escuelas que re- } \\
\text { ciben población de nivel socioeconómico desfavorable. } \\
\text { Los supervisores fiscalizan las subvenciones en los centros educati- } \\
\text { vos subvencionados, controlan el cumplimiento de la normativa vi- } \\
\text { gente y promueven la autoevaluación escolar. } \\
\text { La supervisión administrativa está en manos de otros agentes. } \\
\text { La evaluación externa de los aprendizajes la realiza el Sistema de } \\
\text { Medición de Calidad de la Educación. } \\
\text { Organización cercana a las escuelas, con rasgos de NGP. }\end{array}$ \\
\hline $\begin{array}{l}\text { Con } \\
\text { asiento } \\
\text { en la } \\
\text { escuela }\end{array}$ & Finlandia & $\begin{array}{l}\text { La Inspección se suprimió en 1991, apostando a la confianza en la } \\
\text { libertad pedagógica de los docentes y en su formación profesional, } \\
\text { así como en la autoevaluación institucional, en la que participan ac- } \\
\text { tores institucionales, locales y padres. } \\
\text { En } 2014 \text { comenzó un sistema de evaluación externa a cargo de la } \\
\text { agencia gubernamental Finish Education Evaluation Center. } \\
\text { Organización intermedia, con asiento en las escuelas. }\end{array}$ \\
\hline
\end{tabular}

Fuente: Elaboración propia con base en la bibliografía referida.

Desde una perspectiva institucionalista (North, 1995), los modelos de supervisión referidos constituyen las reglas de juego vigentes para los inspectores, aun cuando el 
posicionamiento de los actores nunca está determinado exclusivamente por la institucionalidad, como señalaron hace tres décadas Crozier y Friedberg (1990):

Las estructuras y las reglas que rigen el funcionamiento oficial de una organización [...] crean y circunscriben «zonas organizadas de incertidumbre» que los individuos o los grupos tratarán simplemente de controlar para utilizarlas en la consecución de sus propias estrategias y alrededor de las cuales se crearán, por ende, relaciones de poder. (P. 66)

\section{El cambio educativo}

El estudio del cambio educativo tiene una larga trayectoria. Fullan (1993) desarrolló un enfoque de la complejidad de los procesos de cambio postulando que estos no son lineales, ya que los individuos y los grupos de una organización compleja, como la escuela, reflejan una particular sociedad, también compleja. Escuela y sociedad solo pueden aprender y luchar juntos por cambiar las condiciones de aprendizaje de sus alumnos y de sí mismos. Estas son las change forces o capacidades internas y externas de aprendizaje organizacional.

Fullan y Hargreaves (2006) conceptualizaron la incidencia de dos variables en el cambio de los procesos de enseñanza y de aprendizaje: el maestro en su complejidad, en su carrera y ciclo vital, y la cultura del centro educativo. Proponen caracterizar la cultura de trabajo docente en las conocidas cuatro categorías: individual, balcanizada, de colegialidad forzada y colaborativa.

Por su parte, Vogliotti y Macchiarola (2003) plantean que un cambio educativo no se produce en forma espontánea ni casual, sino intencional y deliberadamente. Las transformaciones deben impactar en dos niveles: 1) el objetivo, que incluye las prácticas docentes, los contenidos de enseñanza, las estrategias metodológicas, los materiales curriculares y formas de encarar la diversidad, los enfoques y prácticas de evaluación y las culturas de trabajo; 2) el subjetivo, vinculado a las representaciones y creencias desde las cuales se interpretan y llevan adelante las innovaciones. Sin estas transformaciones subjetivas no ocurren las objetivas.

Por último, en este artículo se apela al institucionalismo histórico (IH) como una lente interpretativa densa y potente, de la cual se rescata específicamente el concepto de dependencia de la trayectoria: una vez que una institución es adoptada, comienza a transitar etapas de autorreforzamiento que la vuelven más estable y difícil de modificar (North, 1995; Pierson, 1994; Evans et al., 1985). 


\section{Metodología}

Este artículo constituye un estudio de caso; concretamente, el de la Inspección Docente de Uruguay en el período 2005-2020. El enfoque es descriptivo, comparativo y explicativo. Primero, se caracterizan las inspecciones de Primaria y Secundaria en cuanto a su organización y funcionamiento. Segundo, se analiza el modelo uruguayo a la luz de la tipología de De Grauwe y Carron (2003), ajustada por las autoras. Tercero, se explica el freno al cambio en Secundaria y el impuso en Primaria con base en factores pedagógicos y politológicos.

La técnica de relevamiento principal empleada fue la entrevista semiestructurada: se realizaron 21 entrevistas a partir de una muestra finalística definida en función de dos criterios teóricos: el ciclo educativo de los supervisores (primaria y secundaria) y la participación en innovaciones (involucrados y no involucrados). En este sentido, se entrevistó a cinco inspectores de primaria involucrados en innovaciones y siete no involucrados, mientras que de secundaria fueron cinco los participantes en las innovaciones y cuatro los no participantes.

El instrumento de estas entrevistas indagó respecto al papel del entrevistado en relación con el programa innovador, las dificultades encontradas en su implementación, el nivel de apropiación percibido en los docentes y directores, las fortalezas y debilidades del programa, su posicionamiento ante la innovación y finalmente el apoyo político recibido de las autoridades.

\section{Resultados}

\section{La organización y las funciones de la supervisión en Uruguay}

El gobierno de la educación uruguaya ha sido históricamente peculiar. La Administración Nacional de Educación Pública (ANEP) es el organismo responsable de planificar, gestionar y administrar el sistema educativo en educación inicial, primaria, secundaria, técnica y en la formación docente, y goza de autonomía respecto al Poder Ejecutivo. El organismo está encabezado por el Consejo Directivo Central (CODICEN), de cinco miembros. Hasta la aprobación de la Ley de Urgente Consideración (Uruguay, 2020), en 2020 dependían del CODICEN cuatro consejos desconcentrados, cada uno compuesto por tres miembros, que dirigían los distintos niveles educativos: el Consejo de Educación Inicial y Primaria (CEIP), el Consejo de Secundaria (CES), el Consejo de Educación Técnico-Profesional (CETP) y el Consejo de Formación en Educación (CFE, encargado de la formación docente). 
Este artículo estudia las inspecciones del CEIP y el CES, que presentan como rasgo común su carácter weberiano: son burocracias estatales, profesionales, con una carrera definida de forma clara en el Estatuto del Funcionario Docente (EFD), obedecen a su autoridad jerárquica y actúan según los procedimientos administrativos establecidos.

Ambas inspecciones están conformadas por supervisores que tienen diversos grados jerárquicos según el Escalafón Docente, con funciones, responsabilidades y remuneraciones diferentes. Tanto en el CES como en el CEIP, los supervisores califican a los docentes: en el CEIP, los inspectores califican a los directores y los maestros; en el CES, los inspectores de Institutos y Liceos califican a los directores, y los inspectores de cada asignatura, a los profesores que dictan dicha asignatura. Las dos inspecciones supervisan las dimensiones pedagógica, organizacional y administrativa de los centros educativos, pero no califican su desempeño integral como organizaciones educativas, lo cual constituye una omisión crítica en el sistema de evaluación uruguayo.

La Inspección del CEIP está integrada por 288 inspectores y la del CES por 74, entre inspectores de Institutos y Liceos e inspectores de Asignatura. ${ }^{5}$ Esta diferencia numérica es importante en un contexto en el cual el número de docentes es similar en ambos subsistemas: 23.390 y 21.959 respectivamente (Administración Nacional de Educación Pública, 2020).

Un segundo rasgo diferencial es que Primaria cuenta con inspectores en todos los grados del Escalafón, mientras que en Secundaria prácticamente no hay inspectores de alto grado (V y VI); ${ }^{6}$ la pirámide organizacional es entonces más achatada en Secundaria que en Primaria. Adicionalmente, en Primaria existen 215 supervisores cercanos a la escuela (los llamados inspectores de zona), mientras que en Secundaria esta figura no existe.

En tercer término, el CEIP posee una larga tradición de regionalización de la Inspección, con inspectores departamentales y regionales, pero la descentralización de la supervisión en Secundaria es reciente e incipiente. Recién en el 2014 el CES aprobó la creación de inspectores coordinadores regionales, con jerarquía sobre los inspectores de Institutos y Liceos, pero los inspectores de asignaturas mantienen un alcance nacional.

La descripción de la Inspección de Primaria y Secundaria en Uruguay muestra que su organización y sus funciones son similares a las de Francia y España, y corresponden al tipo ideal de control público (tabla 1): la organización es jerárquica; son los inspectores quienes evalúan y califican a los maestros y profesores; cada docente tiene derecho a ser supervisado periódicamente; los docentes se sienten responsables ante la autori-

5 Información correspondiente al año 2019.

6 Por acta 56, resolución 60, del 2 de diciembre de 2020, el CES suplió el cargo de inspector general, que permanecía vacante desde el 30 de marzo de 2019. 
dad educativa que los contrata (CEIP y CES), más que frente a los directores de los centros educativos; como en España, se creó un Instituto Nacional de Evaluación Educativa (INEE en España, INEEd en Uruguay).

Múltiples son las normas y los dispositivos que alejan el caso uruguayo de los otros tres tipos de la referida tipología. En Uruguay la Inspección no cumple las funciones de evaluar las escuelas como tales ni de establecer parámetros de calidad a través de sistemas de indicadores, como sí ocurre en Inglaterra, Nueva Zelanda o Finlandia. A diferencia de Inglaterra o Nueva Zelanda (modelo de control central), las visitas a los centros no son cada tres o cinco años, sino que tienden a ser anuales; el discurso educativo rara vez refiere al principio de eficacia; la noción de accountability no forma parte ni del discurso ni de las prácticas de los actores educativos; no se promueven las autoevaluaciones institucionales; tampoco se contratan evaluadores externos de los centros (como en el caso neozelandés). Respecto a Chile, en Uruguay no se impulsa la autoevaluación institucional; la supervisión no está vinculada en lo más mínimo a subvenciones escolares; la supervisión administrativa es responsabilidad de funcionarios públicos como los inspectores, no de otros agentes contratados como en Chile. Por último, con relación a Finlandia (modelo con asiento en la escuela), Uruguay se ubica en las antípodas de la supresión de la Inspección y la autoevaluación institucional.

Si consideramos la Inspección como unidad de la administración estatal, la uruguaya está en el polo burocrático-tradicional, por la importancia de las jerarquías, el privilegio extremo asignado a la legalidad de los procedimientos administrativos - que desplaza incluso la actuación frente a los requerimientos de las comunidades educativas- y la adopción no cuestionada de la cultura del expediente. En el polo opuesto del continuo se encuentran los casos de Nueva Zelanda - exponente más nítido de la Nueva Gestión Pública (Vicher, 2007)—, Inglaterra (heredera de las reformas de la era Thatcher) y Chile (con los legados de la reforma educativa de Pinochet). En estos países la educación no ha escapado a la flexibilización de los procesos administrativos y se han creado múltiples indicadores y medidas de rendimiento de los resultados. Finalmente, Finlandia podría ser ubicada en una posición intermedia en este continuo.

\section{Los inspectores ante las innovaciones educativas en Uruguay (2005-2020)}

En este apartado se describe el papel de los inspectores frente al cambio educativo en tres dimensiones: su grado de participación en las innovaciones, su posicionamiento frente a ellas y su nivel de apropiación de tales programas.

Toda política pública atraviesa el llamado ciclo de las políticas públicas, desde la inclusión en la agenda hasta la evaluación (Sabatier, 2007). Corresponde preguntarse 
entonces cuál fue el accionar de la Inspección en el ciclo de las innovaciones del período en estudio. De acuerdo a las entrevistas realizadas, la participación de los inspectores fue sustancialmente diferente en Primaria y en Secundaria: en el CEIP los supervisores mayoritariamente consideran que tuvieron un rol activo en el desarrollo de las innovaciones, particularmente durante la implementación y el monitoreo. En contraposición, en el CES los inspectores declaran que su participación fue mínima en todas las fases del ciclo (tabla 2).

A la hora de evaluar el posicionamiento de los inspectores frente a las innovaciones, los entrevistados de Primaria declararon contundentemente que había sido «favorable», mientras que los de Secundaria manifestaron que había sido «neutro» porque en varias ocasiones una parte de la Inspección había adoptado una postura favorable mientras que otra se había opuesto rotundamente a la innovación (tabla 3).

Concomitantemente, en el CEIP la apropiación de los cambios por los inspectores, directores y docentes de aula fue bastante más profunda que en el CES: a juzgar por las entrevistas, en Secundaria la apropiación fue media y baja, en tanto en Primaria fue alta (tabla 4).

Tabla 2. Opinión de los entrevistados sobre la participación de la Inspección en el ciclo de las innovaciones en Primaria y Secundaria. Uruguay, 2005-2020. En frecuencias

\begin{tabular}{|l|c|c|}
\hline \multirow{2}{*}{ Nivel educativo } & \multicolumn{2}{|c|}{ Participación } \\
\cline { 2 - 4 } & \multicolumn{2}{|c|}{ No participó } \\
\hline Primaria & 5 & 7 \\
\hline Diseño & 12 & 0 \\
\hline Implementación & 12 & 0 \\
\hline Monitoreo & 3 & 9 \\
\hline Evaluación & & 9 \\
\hline Secundaria & 0 & 6 \\
\hline Diseño & 3 & 7 \\
\hline Implementación & 2 & 9 \\
\hline Monitoreo & 0 & \multicolumn{2}{|c|}{} \\
\hline Evaluación & & \\
\hline
\end{tabular}

Preguntas: ¿Qué papel tuvo la Inspección en el diseño del programa? ¿Cuál fue el rol de la Inspección en su implementación? Los inspectores, ¿monitorearon (dieron seguimiento) y/o evaluaron el Programa? 
Fuente: Elaboración propia.

Tabla 3. Opinión de los entrevistados respecto al posicionamiento de la Inspección frente a las innovaciones en Primaria y Secundaria. Uruguay, 2005-2020.

En frecuencias

\begin{tabular}{|l|c|c|c|}
\hline \multirow{2}{*}{ Nivel educativo } & \multicolumn{3}{|c|}{ Posicionamiento } \\
\cline { 2 - 4 } & Favorable & Neutro & Desfavorable \\
\hline Primaria & 8 & 3 & 1 \\
\hline Secundaria & 2 & 5 & 2 \\
\hline
\end{tabular}

Pregunta: Si pensamos en la Inspección en su conjunto, ¿cómo ve usted su posicionamiento (favorable, neutro, no favorable) ante el programa?

Fuente: Elaboración propia.

Tabla 4. Opinión de los entrevistados respecto al nivel de apropiación de las innovaciones por inspectores, directores y maestros en Primaria y Secundaria. Uruguay, 2005-2020. En frecuencias

\begin{tabular}{|c|c|c|c|c|}
\hline \multirow[t]{2}{*}{ Nivel educativo } & \multicolumn{4}{|c|}{ Nivel de apropiación } \\
\hline & Alto & Medio & Bajo & Total \\
\hline \multicolumn{5}{|l|}{ Primaria } \\
\hline Inspectores & 7 & 5 & 0 & 12 \\
\hline Directores & 8 & 4 & 0 & 12 \\
\hline Maestros & 8 & 4 & 0 & 12 \\
\hline Total de menciones & 23 & 13 & 0 & - \\
\hline \multicolumn{5}{|l|}{ Secundaria } \\
\hline Inspectores de Institutos y Liceos & 0 & 4 & 5 & 9 \\
\hline Inspectores de asignaturas & 0 & 5 & 4 & 9 \\
\hline Directores & 3 & 3 & 3 & 9 \\
\hline Profesores & 0 & 2 & 7 & 9 \\
\hline Total de menciones & 3 & 14 & 19 & - \\
\hline
\end{tabular}


Preguntas: Para usted, ¿cuál fue el nivel de apropiación (conocimiento, adhesión) de los inspectores del Programa? ¿Y de los directores? ¿Y de los docentes?

Fuente: Elaboración propia.

\section{¿Cómo explicar el impulso al cambio en Primaria y el freno en Secundaria?}

Las instituciones importan. Así lo ha mostrado el institucionalismo en sus diversas vertientes. Sin embargo, en el marco de una institucionalidad similar, los inspectores de Primaria tendieron a impulsar los cambios, mientras que los de Secundaria se inclinaron hacia la resistencia, con excepciones. Este apartado plantea qué factores incidieron sobre estas posturas diferenciales.

Un primer elemento explicativo es el apoyo político dado a las innovaciones por las autoridades educativas. Las percepciones de los entrevistados de Primaria se concentraron en la categoría apoyo político alto, no así las de los entrevistados de Secundaria, que se dividieron en alto, medio e incluso bajo (tabla 5). Ello resulta llamativo si se toma en cuenta que las transformaciones consideradas fueron creadas por tres gobiernos del mismo partido, el Frente Amplio.

Tabla 5. Opinión de los entrevistados respecto al apoyo político a las innovaciones en Primaria y Secundaria. Uruguay, 2005-2020. En frecuencias

\begin{tabular}{|l|c|c|c|}
\hline \multirow{2}{*}{ Nivel educativo } & \multicolumn{3}{|c|}{ Nivel de apoyo político } \\
\cline { 2 - 4 } & Alto & Medio & Bajo \\
\hline Primaria & 11 & 1 & - \\
\hline Secundaria & 3 & 4 & 2 \\
\hline
\end{tabular}

Pregunta: ¿Qué nivel de apoyo político (autoridades de ANEP) tuvo el programa? Fuente: Elaboración propia.

Un segundo factor reside en la cultura profesional de los dos organismos; esto es, el conjunto de creencias, expectativas, representaciones, hábitos y maneras de actuar de las distintas comunidades educativas. Tal cultura profesional genera una personalidad particular, un ethos o valores básicos que cada colectivo docente construye a partir de sus procesos de socialización profesional y de las relaciones entre sus integrantes (Fullan y Hargreaves, 2006). Los testimonios relevados muestran la existencia de dos culturas profesionales divergentes en Primaria y Secundaria.

Vogliotti y Macchiarola (2003), así como Fullan y Hargreaves (2006), subrayan la importancia de las modalidades de implementación del cambio educativo (tabla 6). Los primeros distinguen entre los factores objetivos y los subjetivos de las innovaciones. 
Los segundos refieren al trabajo docente y la cultura del centro educativo. En tal sentido, los entrevistados de Secundaria expresaron que, más allá del buen diseño de los programas, la dinámica liceal operó como un obstáculo cultural insuperable, sea por la falta de liderazgo de la dirección, la alta rotación del personal docente, la estructura de la carrera docente y/o la escasa participación en las innovaciones.

Tabla 6. Factores explicativos del papel de los Inspectores en el cambio educativo en Primaria y Secundaria. Uruguay, 2005-2020

\begin{tabular}{|l|c|c|}
\hline Criterios & Primaria & Secundaria \\
\hline Fullan y Hargreaves (2006) & 6 & 9 \\
\hline Docente en su complejidad, carrera y ciclo vital & 6 & 9 \\
\hline Cultura del centro educativo & \multicolumn{2}{|l|}{} \\
\hline Fullan y Hargreaves (2006) & 0 & 7 \\
\hline Aplicación sin involucramiento docente & 8 & 2 \\
\hline Aplicación con involucramiento docente & 4 & 9 \\
\hline Procesos complejos de cambio & & 0 \\
\hline Vogliotti y Macchiarola (2003) & 5 & 7 \\
\hline Factores objetivos & & \\
\hline Factores subjetivos & 7 & \\
\hline
\end{tabular}

Fuente: Elaboración propia.

Finalmente, un cuarto elemento que permite dilucidar por qué en Primaria predominó la promoción del cambio y en Secundaria el bloqueo remite al peso de la historia, el path dependence en la terminología de los institucionalistas (North, 1995).

Históricamente, los maestros primarios y los profesores secundarios transitaron por una formación inicial diferencial y construyeron culturas de trabajo disímiles. Los maestros primarios poseen una formación más generalista y su objetivo pedagógico primordial es la formación integral del niño; a su vez, en el ejercicio de su práctica, integran equipos docentes significativamente más pequeños que los de Secundaria. En contraposición, la creación de enseñanza secundaria se relaciona con la preparación para la educación universitaria, lo que dejó una profunda huella en los currículos, que hoy siguen siendo asignaturistas y fragmentados. 
Estos elementos permean las culturas profesionales de ambas inspecciones. En Primaria prevalece una mayor cohesión, esquemas de interpretación compartidos y actuaciones más articuladas, mientras que en Secundaria se tiende a la balcanización, lo cual conspira contra una articulación sólida y lleva al colectivo a comportarse como una anarquía organizada (Morgan, 1990).

\section{Discusión y conclusiones}

El análisis del caso uruguayo ilustra respecto a los modelos de supervisión identificados en el escenario internacional. A través de un enfoque comparativo se profundiza en el papel de la inspección respecto al cambio educativo. Esto es relevante en cuanto la literatura especializada no ha abordado el rol de mediación de los inspectores en las innovaciones. Adicionalmente se aporta una explicación novedosa en la que se conjugan el acervo proveniente de la pedagogía con el ligado al estudio de las políticas educativas.

Tanto los modelos de supervisión educativa como los de administración pública constituyen las reglas de juego que rigen el comportamiento de los actores. Sin embargo, estos siempre encuentran zonas de incertidumbre entre las que desarrollan su accionar.

Con relación a las preguntas que guían esta investigación, surge que, bajo la misma institucionalidad, en Uruguay en el período 2005-2020 la postura de la Inspección de Primaria ante las innovaciones fue abierta, muy diferente a la asumida por la Inspección de Secundaria. La pregunta es por qué y la respuesta conlleva la necesidad de complementar la variable institucional con otras que expliquen el bloqueo al cambio educativo por la Inspección de Secundaria y el estímulo por la de Primaria.

El caso uruguayo revela que el papel de la supervisión en relación con la transformación educativa deriva no solo de la institucionalidad dominante, sino también del respaldo político, la modalidad de implementación de las políticas innovadoras, la cultura profesional prevaleciente y el peso de la historia.

Respecto al primer factor, las entrevistas revelaron que los inspectores de Primaria percibieron un permanente apoyo político a las innovaciones por parte de las autoridades. Las de Secundaria, por el contrario, oscilaron en ese respaldo a lo largo del tiempo. A título de ejemplo, el Programa de Aulas Comunitarias recibió un fuerte respaldo político durante el período 2005-2010, un respaldo medio entre los años 2010 y 2015, y careció de él a partir de entonces.

En cuanto a la cultura profesional prevaleciente, la información permite perfilar una cultura balcanizada en Secundaria, frente a elementos de una cultura colaborativa y disciplinada en Primaria. 
La modalidad de ejecución del cambio emerge como el cuarto factor explicativo de las diferencias entre Primaria y Secundaria: en la Inspección de Secundaria pesaron significativamente el carácter top-down de la implementación, la falta de participación de los inspectores en su conceptualización, así como el no reconocimiento de la complejidad que conlleva toda transformación educativa.

Finalmente, la historia pesó en la consecución o la frustración de las innovaciones. La historia de Primaria se remonta a fines del siglo XIX; la de Secundaria, a los años treinta del siglo XX, cuando se separó de la Universidad. Por décadas los maestros primarios se formaron como docentes generalistas, mientras que los profesores secundarios mantuvieron la especialización disciplinaria. Esta diversidad de formaciones se unió a condiciones de trabajo diferentes: los maestros trabajan en escuelas de poco personal, con compañeros a los que conocen y con los que dialogan cotidianamente; en contraposición, los docentes de Secundaria son profesores taxi, que actúan en varios liceos y se encuentran con muchos colegas a quienes apenas llegan a conocer. La comunicación, el diálogo, la creación de los factores subjetivos que dan cabida a la innovación son bastante más difíciles de gestar en los liceos que en las escuelas.

En síntesis, para concretar el cambio educativo no alcanza con alterar las reglas de juego; es necesario trabajar arduamente con los actores en las dimensiones política, técnica y cultural.

\section{Referencias}

Administración Nacional de Educación Pública. (2020). Censo nacional docente. Montevideo: ANEP. Recuperado de https://censodocente2018.anep.edu.uy/censo/.

Aguerrondo, I. (2013). El rol de la supervisión educativa en la gestión de las políticas públicas. Educar, 49(1), 13-27. doi: https://doi.org/10.5565/rev/educar.

Bentancur, N., y Mancebo, M. E. (2011). La educación en la encrucijada: Escrutinio político y gobierno de la enseñanza. En La política en tiempos de Mujica: En busca del rumbo. Montevideo: Facultad de Ciencias Sociales. (Informe de Coyuntura, 10).

Bueno, G., y Cababaro, D. (2019). Supervisory practices in an autonomous national high school: Teachers'cross-sectional perspectives. Ponencia presentada en Philippines Int'l Conference on Economics, Education, Humanities \& Social Sciences, Cebu. doi: https://doi.org/10.17758/ERPUB3.UH0119419.

Canal Ventosa, I. (2018). Estudio comparativo de la evaluación al profesorado en Reino Unido, Finlandia y España (Tesis de maestría). Universidad de Cantabria, Santander. 
Choi, A. (2019). La inspección de la educación: ¿Qué modelos funcionan mejor? Evidencias para la mejora educativa. Barcelona: Institut Catalá d'Avaluació de Politiques Públiques. Recuperado de https://www.fbofill.cat/sites/default/files/Que_funciona_13_inspeccioneducativa.pdf.

Crozier, M., y Friedberg, E. (1990). El actor y el sistema: Las restricciones de la acción colectiva. México: Alianza Editorial Mexicana.

Grauwe, A. de, y Carron, G. (2003). Tendencias en supervisión escolar: Cuestiones de actualidad en supervisión. Una revisión de la literatura. París: IIPE-UNESCO.

Evans, P., Rueschemeyer, D., y Skocpol, T. (1985). Bringing the state back in. Nueva York: Cambridge University Press.

Fernández, T., y Mancebo, M. E. (2015). Las políticas de inclusión educativa en Uruguay: ¿Erosión de la matriz institucional clásica de la arena educativa? Revista Latinoamericana de Políticas y Administración de la Educación, (3), 71-89.

Fernández, T., y Pereda, C. (2010). Panorama de las políticas de inclusión educativa en la educación media y superior (2005-2009). En T. Fernández (comp.), La desafiliación en la educación media y superior de Uruguay: Conceptos, estudios y políticas. Montevideo: Universidad de la República.

Fullan, M. (1993). Change forces: Probing the depths of educational reform. Londres: Routledge Falmer.

Fullan, M., y Hargreaves, A. (2006). La escuela que queremos: Los objetivos por los cuales vale la pena luchar. Buenos Aires: Amorrortu.

Instituto Nacional de Evaluación Educativa. (2014). Informe sobre el estado de la educación. Montevideo: INEEd.

Mancebo, M. E., y Pérez, J. (2018). Del dicho al hecho hay un gran trecho: Obstáculos para la expansión de la escolaridad media en Uruguay. Archivos Analíticos de Políticas Educativas, 26(161), 1-29. doi: 10.14507/epaa.26.3189.

Marshall, K. (2005). It's time to rethink teacher supervision and evaluation. Phi Delta Kappan, 86(10), 727-735. doi: 10.1177/003172170508601004.

Moreno, M., Rojo, V., Chiossi, J., Evans, T., González, M., y Olivera, L. (2014). Informe de evaluación del Programa Aulas Comunitarias (PAC). Montevideo: MIDES.

Morgan, G. (1990). Imágenes de la organización. Madrid: RA-MA.

North, D. (1995). Instituciones, cambio institucional y desempeño económico. México: Fondo de Cultura Económica.

Pérez, J. (2011). Los programas de inclusión educativa en el marco del Plan de Equidad: Un estudio sobre la coordinación de políticas públicas (Tesis de grado). Universidad de la República, Montevideo.

Pierson, P. (1994). Dismantling the Welfare State? Reagan, Thatcher and the politics of retrenchment. Londres: Cambridge University Press. 
Rivera Hernández, C. (2017). Propuesta de un sistema de formación especializada en supervisión educativa: Análisis comparativo de 13 sistemas de supervisión escolar del mundo. Innovación educativa (México, DF), 17(74), 165-179.

Romero, M. (2018). Entre el mandato inclusor y la realidad: Incidencia de las representaciones sociales de los docentes en la construcción de culturas inclusivas (Tesis doctoral). Universidad ORT, Montevideo.

Romero, M. (2011). Desafíos de la educación media: Inclusión y calidad educativa. Páginas de Educación, 4(1). doi: 10.22235/pe.v4i1.634.

Sabatier, P. (2007). Theories of the policy process. Cambridge: Westview Press.

Sergiovanni, T., y Starratt, R. (2007). Supervision: A redefinition. Boston: McGraw-Hill.

Sistema de Información de Tendencias Educativas en América Latina. (2020). Uruguay. Recuperado de https://siteal.iiep.unesco.org/pais/uruguay.

United Nations Educational, Scientific, and Cultural Organization. (2021). Uruguay. Recuperado de http://uis.unesco.org/en/country/uy.

Uruguay. (2020, 14 de julio). Ley 19.889: Ley de Urgente Consideración. Recuperado de https://legislativo.parlamento.gub.uy/temporales/docu7957274277353.htm.

Vicher, D. (2007). La reforma neogerencial en Nueva Zelanda. Convergencia, 14(4), 163186. Recuperado de http://www.scielo.org.mx/scielo.php?script=sci_arttext\& pid=S1405-14352007000300007.

Vogliotti, A., y Macchiarola, V. (2003). Teorías implícitas, innovaciones educativas y formación profesional de los docentes. Córdoba: Universidad Nacional de Río Cuarto.

Zorrilla, M. (2013). Transformar la supervisión escolar: ¿Solo una aspiración o puede ser una meta razonable y posible? Educar, 49(1), 49-46. doi: 10.5565/rev/ educar.10. 